\title{
Early misdiagnosis of acute aortic dissection: A case report and analysis of the causes
}

\author{
Xiao-yan Chen, Fan-liang Kong, Tong-guo Wu* \\ Department of Cardiology, Guangzhou Red Cross Hospital, Medical College of Jinan University, Guangzhou, Guangdong, China
}

Received: August 27, 2016

Accepted: September 22, $2016 \quad$ Online Published: October 23, 2016

DOI: $10.5430 /$ ijdi.v4n1p15

URL: http://dx.doi.org/10.5430/ijdi.v4n1p15

\begin{abstract}
Type A aortic dissection is a catastrophic clinical entity involving the ascending aorta. In this case report, a patient was admitted to the emergency room with a presentation resembling acute myocardial infarction (AMI) that led to the inappropriate administration of anticoagulant agents or platelet. This is a case report of a 69-year-old male patient with early misdiagnosis and analysis of type A aortic dissection with discussion on the causes of misdiagnosis in light of the literature.
\end{abstract}

Key Words: Acute aortic dissection, Acute myocardial infarction, Misdiagnosis, Aortic intramural hematoma

\section{INTRODUCTION}

Type A dissection refers to involvement of ascending aorta, while type B refers to the situation where ascending aorta is spared from involvement. Acute type A aortic dissection is a severe and sophisticated disease that requires immediate diagnosis. When helical computed tomography (CT), magnetic resonance imaging (MRI) and other imaging tests to the pathology are not obvious, acute aortic dissection is very easy to be misdiagnosed as acute myocardial infarction that is another fatal vascular emergency requiring early diagnosis and intervention. Overall in-hospital mortality for Type A dissection is around 30\%. Delay in diagnosis increases the hourly mortality rate by $1 \% .^{[1]}$

CT with higher sensitivity and specificity, is the preferred imaging test in the diagnosis of suspecting AAD and its accuracy has been improved by the recent availability of ultrafast scanning, large scanning range and three-dimensional reconstruction. MRI offers improved anatomic delineation of the aorta and can provide high-quality images in several planes, therefore its correct diagnosis rate is higher. Echocardiography can be displayed the aortic from root to arch through the left ventricular long axis view et al, and can be done in the bedside, which improve the diagnostic rate of the ascending aortic root dissection as quickly as possible.

In this case, a 69-year-old male patient was presented to the emergency department with complaints of chest pain. He had syncope twice. He was inappropriately admitted to the anticoagulant agents and platelet service with misdiagnosis of AMI, however, further evaluation and analysis revealed a Type A aortic dissection 5 days later.

\section{Case Presentation}

A 69-year-old, 70-kilogram, male patient was presented to the emergency department with complaining of severe chest pain for 6 days. He suffered extreme pain in the last 17 hours. He also had syncope twice each lasting about 5 minutes causing him to wake up and this prompted him to admit himself to hospital. He appeared pale, diaphoretic and in extreme

\footnotetext{
*Correspondence: Tong-guo Wu; Email: wutongguo@sohu.com; Address: Department of Cardiology, Guangzhou Red Cross Hospital, Medical College of Jinan University, Guangzhou 510220, Guangdong, China.
} 
distress. He had no previous known medical or surgical conditions nor history of smoking and drinking. He also had no regular physical examination.

A physical examination revealed that his blood pressure was 90/52 mmHg using dopamine maintenance intravenous micro pump and heart rate 120 beats/min. He was conscious, cooperative and breathing slightly. His jugular was impeded with fluid. His lungs did not show any moist rales. He had orderly rhythm, and had no audible murmurs or rubs. The complete blood count showed WBC count of $16.05 \times 10^{9} / \mathrm{L}$, HB count of $125 \mathrm{~g} / \mathrm{L}$, PTL count of $47 \times 10^{9} / \mathrm{L}$. The D-dimer level was $3,890 \mu \mathrm{g} / \mathrm{L}$, serum creatinine was $306 \mu \mathrm{mol} / \mathrm{L}$, CTnT was $5.86 \mu \mathrm{g} / \mathrm{L}$ and CK-Mb was $266.5 \mathrm{U} / \mathrm{L}$. Electrocardiogram showed sinus tachycardia and ST-T change was not obvious. AMI was suspected in the admission diagnosis. Other biochemical analysis were normal. His bedside echocardiography showed ventricular wall motion abnormalities, decreased of left ventricular systolic function and a slight of pericardial effusion under the disturbance of lung. There was no obvious pathology except for moderate stenosis at the open of the left clavicle in the thoracoabdominal computed tomography and magnetic resonance angiography. There is no implementation of coronary angiography as the use of nephrotoxic contrast agents will further damage to the renal. Then Aspirin $100 \mathrm{mg}$ and clopidogrel $75 \mathrm{mg}$ plus low molecular weight heparin were administered for treatment.

The patient developed abdominal pain accompanied with shortness of breath, hypoxia (the value of $\mathrm{SpO}_{2}$ [peripheral capillary oxygen saturation] was $88 \%-93 \%$ on $41 \%$ inspired oxygen concentration) on the second day. The biochemical analysis showed severe elevation of ALT (1,260 U/L) and AST (1,795 U/L). The amylase and lipase were $965 \mathrm{U} / \mathrm{L}$ and 37 U/L respectively. Then the patient was treated in the intensive care unit for multiple organ failure. Repeated review of HB and PLT were lower than normal. The lowest PLT level was $48 \times 10^{9} / \mathrm{L}$ and the lowest HB level was $99 \mathrm{~g} / \mathrm{L}$. The patient improved after treatment with anticoagulation, antiplatelet, liver-protecting therapy and myocardial nutrition. He was transferred to the coronary care unit on the 5th day for stable vital signs.

Based on the patient's clinical symptoms, with D-dimer level, troponin I and myocardial enzyme, a decrease in platelet count, no dynamic change in ECG, the images of thoracoabdominal CT and MR (see Figures 1-3), experienced clinicians considered there is high chance of aortic dissection. With a withdrawal of anticoagulation and antiplatelet drugs and a strict control of blood pressure and heart rate, the patient was transferred to the general ward after his condition was stable. Sixteen days after hospitalization, review of echocardiography (see Figure 4) indicated that aorta widened dissection (aortic intramural hematoma formation). In this case, patient was treated with drugs as surgical in the ascending aorta presents specific challenges due to proximity to the aortic root. The patient was uneventfully discharged after 21 days of hospitalization. Aortic intramural hematoma with acute aortic syndrome (A Stanford), Acute pericardial tamponade accompany with acute cardiac shock and acute liver injury in patients with acute renal failure were the discharge diagnosis.

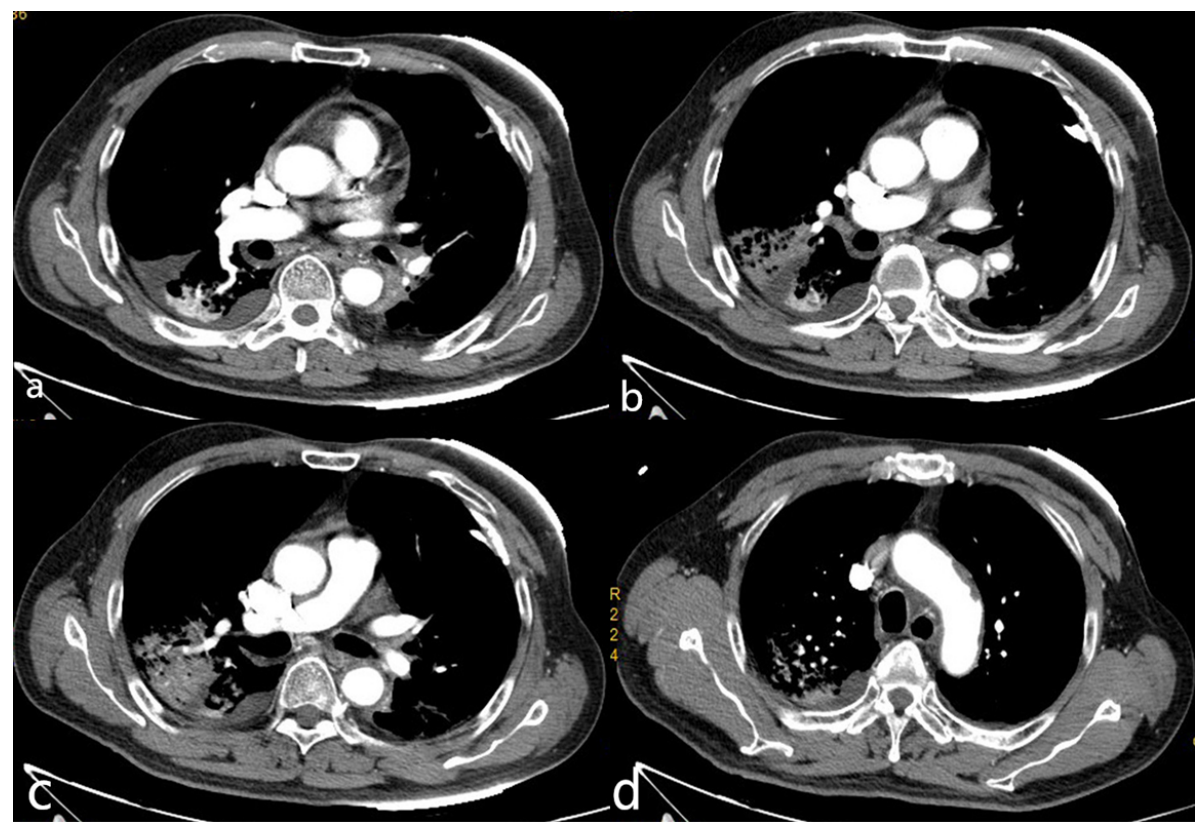

Figure 1. CT showed no obvious pathology in aorta 


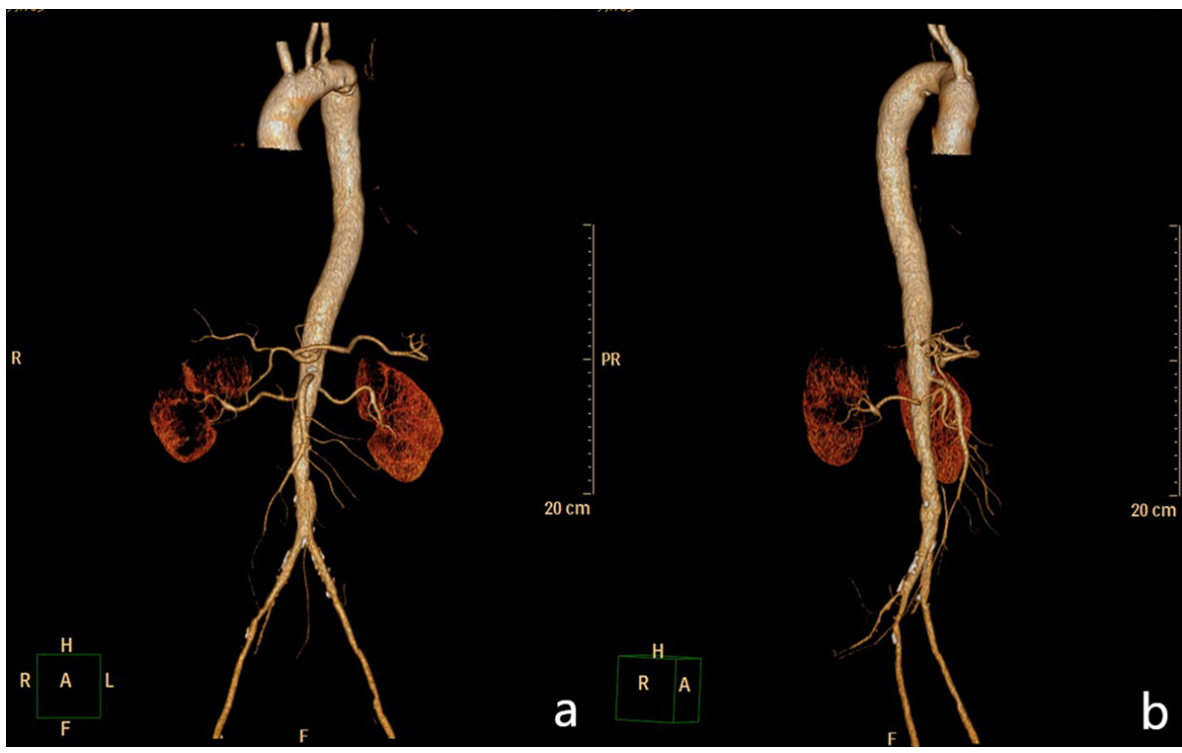

Figure 2. 3D volume rendering CT image showed no obvious pathology in aorta

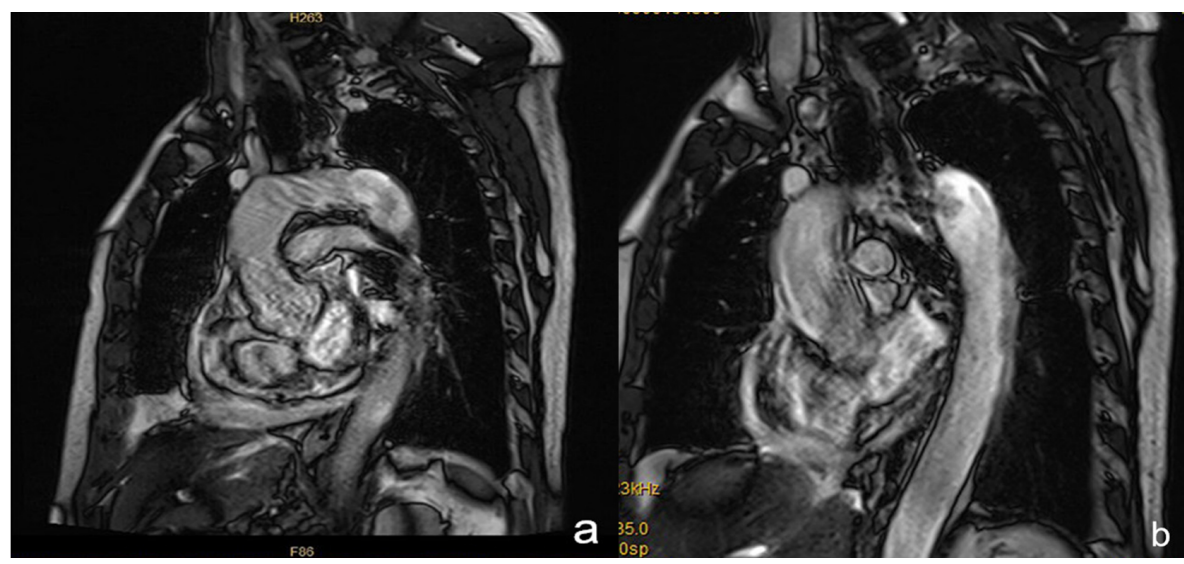

Figure 3. MRA showed no obvious pathology in aorta

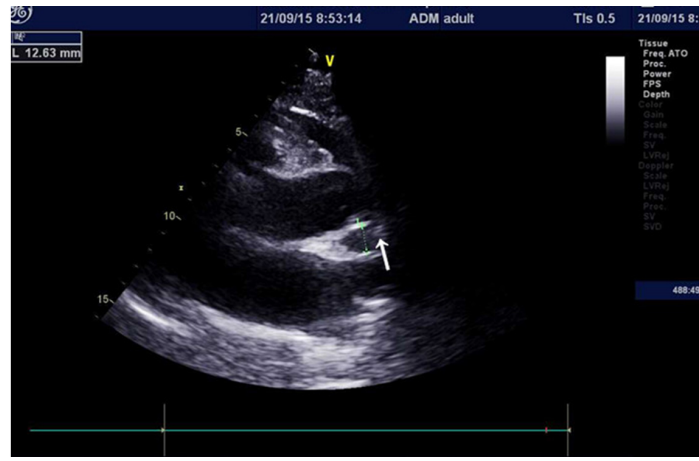

Figure 4. UCG showed that aorta widened dissection (aortic intramural hematoma formation)

\section{Discussion}

Acute aortic dissection (AAD) is a life-threatening disease that requires immediate diagnosis and assessment. Although aortic dissection is an ancient disease, misdiagnosis is still Published by Sciedu Press an open question. This is especially so when imaging tests are not clear in the early diagnosis of disease, which is more likely to be missed or misdiagnosed. Atypical aortic dissection, also known as aortic intramural hematoma, in the past, was thought to be a rare type of aortic dissection, which is rarely diagnosed by radiologists. However, due to the further improvement of the early imaging tests and imaging resolution, the aortic intramural hematoma is not uncommon.

Patients with acute aortic dissection usually complain of severe pain in the chest and hypertension, but can also present a diversed clinical manifestations, such as syncope, anuria, abdominal pain, stroke and limb ischemia. ${ }^{[2]}$ Aortic dissection resembles AMI symptomatically, especially accompanied by myocardial enzyme and CTNT increased, which can lead to early misdiagnosis and inappropriate use of anticoagulation, antiplatelet and thrombolytic therapy or even acute coronary 
angiography and percutaneous coronary intervention immediately. Some of which have been blindly performed with the injection of ventricular and aortic high pressure angiography, leading to disastrous consequences. ${ }^{[3,4]}$

Patients admitted to hospital with this condition often complain of severe chest pains, but the early imaging tests of echocardiography and MR plus CT were untypical in this case. However, CTnT and CK-Mb were significantly increased and clinicians lack of experience, which resulted in misdiagnosis as myocardial infarction early. Myocardial injury markers elevated may be due to the ascending aorta dissection extending to the proximal end, which leads to the aortic valve swelling and tearing, this results in aortic incompetence and coronary artery stenosis at the opening, leading to coronary insufficiency. This case was misdiagnosed as myocardial infarction with inappropriate use of anticoagulation and antiplatelet drugs. It has been reported that early thrombocytopenia is an independent predictor of mortality in aortic dissection. In this case, the patient's platelet count decreased constantly for about a week after admission, which was related to the consumption of platelets and coagulation disorders due to extensive thrombosis. ${ }^{[5]}$ Therefore, clinicians should be alert to the possibility of aortic dissection in patients with the early reduced of platelet count and no dynamic changes in the ECG during the period of hospitalization, even if the markers of myocardial injury were increased. Routine blood tests of aortic dissection usually are not diagnosed, however, in the case of chest pain D-dimer is helpful in differentiating acute myocardial infarction and aortic dissection, especially dynamic elevation being more significant. The latest guidelines of ESC highlight the importance of dynamic monitoring of D-dimer, which is highly elevated in both acute pulmonary embolism (PE) and AAD. ${ }^{[6]}$ Therefore, we should pay attention to identify three common cardiovascular emergencies: AMI, AAD and acute PE for the patients whose D-dimer elevated significantly.

Chest X-ray, helical CT, MRI, transthoracic echocardiogra- phy and transesophageal echocardiography (TEE) are available to evaluate patients with suspected aortic dissection. AAD is a potentially fatal condition that requires rapid assessment and treatment. Chest X-rays may reveal mediastinal widening, abnormality in aortic contour, or pleural effusion in the patients with AD. ${ }^{[7]} \mathrm{CT}$ with higher sensitivity and specificity, is the preferred imaging test in the diagnosis of suspected AAD. ${ }^{[8]}$ It is difficult to monitor patients who have unstable hemodynamics with AAD as the result of MR is requires a longer time, ${ }^{[9]} \mathrm{MR}$, however, is useful for the evaluation of stable and chronic dissection. However echocardiography that can be performed in the bedside or emergency room is more feasible and valuable for imaging diagnostic techniques for the initial evaluation of suspected AAD. But there are reports that imaging findings were completely normal in $12 \%$ of patients with AD5. ${ }^{[5]}$ This is because the heart's activity affects the imaging quality of CTA and MRI, which often causes the missed diagnosis, especially the ascending aortic root dissection, however, experienced specialists of ultrasonic cardiography (UCG) combined with clinical symptoms can help to improve the diagnostic rate of $\mathrm{AD}$, minimizing missed diagnosis or misdiagnosis. ECG gating of the pulmonary artery, coronary artery and aortic sequential CT angiography is helpful in diagnose acute PE, AMI and AAD, which is a positive recommendation, based on the international guidelines, for the diagnosis and treatment of cardiovascular diseases in recent years.

\section{Conclusion}

Clinicians should enhance awareness in the diagnosis of aortic dissection based on clinical symptoms and physical examination when the imaging tests of CT and MR are not typical. Echocardiography is a very important auxiliary diagnosis technology for ascending aortic dissection.

\section{Conflicts of Interest Disclosure}

The authors have declared no conflicts of interest.

\section{REFERENCES}

[1] Salman AE, Çeliksoy M, Yetişir F, et al. The importance of clinical suspicion in the diagnosis of a successfully managed case with De Bakey Type 1 acute aortic dissection: A case report. Ulus Cerrahi Derg. 2013; 30(1): 51-3.

[2] Rogers AM, Hermann LK, Booher AM, et al. Sensitivity of the aortic dissection detection risk score, a novel guideline-based tool for identification of acute aortic dissection at initial presentation: Results from the international registry of acute aortic dissection. Circulation. 2011; 123(20): 2213-8. PMid:21555704 http: //dx.doi .org/10. 1161/CIRCULATIONAHA. 110.988568
[3] Lentini S, Perrotta S. Aortic dissection with concomitant acute myocardial infarction: From diagnosis to management. J Emerg Trauma Shock. 2011; 4(2): 273-8. PMid:21769215 http: //dx.doi.org/1 $0.4103 / 0974-2700.82221$

[4] Butler J, Davies AH, Westaby S. Streptokinase in acute aortic dissection. BMJ. 1990; 300(6723): 517-9. PMid:2107933 http: //dx.doi.org/10.1136/bmj.300.6723.517

[5] Yoon SH, Cho YS, Park CY, et al. Disseminated intravascular coagulation associated with aortic dissecting aneurysm. Korean J Intern Med. 2004; 19(2): 134-6. PMid:15366648 http://dx.doi.org/1 $0.3904 / \mathrm{kj}$ im.2004.19.2.134 
[6] Eggebrecht H, Naber CK, Bruch C, et al. Value of plasma fibrin D-dimers for detection of acute aortic dissection. J Am Coll Cardiol. 2004; 44(4): 804-9. PMid:15312863 http://dx.doi.org/10.10 $16 / j \cdot j$ acc. 2004.04 .053

[7] Von Kodolitsch Y, Nienaber CA, Dieckmann C, et al. Chest radiography for the diagnosis of acute aortic syndrome. Am J Med. 2004; 116(2): 73-7. PMid:14715319 http://dx.doi.org/10.1016/j . amjmed.2003.08.030

[8] Shiga T, Wajima Z, Apfel CC, et al. Diagnostic accuracy of trans- esophageal echocardiography, helical computed tomography, and magnetic resonance imaging for suspected thoracic aortic dissection: systematic review and meta-analysis. Arch Intern Med. 2006; 166(13): 1350-6. PMid:16831999 http://dx.doi.org/10.1001 /archinte.166.13.1350

[9] Nienaber CA, Eagle KA. Aortic Dissection: New frontiers in diagnosis and management. Part I: From Etiology to Diagnostic Strategies. Circulation. 2003; 108(5): 628-35. PMid:12900496 http: //dx.doi.org/10.1161/01.CIR.0000087009.16755.E4 